\title{
Vascular Cell Adhesion Molecule-1 Is Expressed in Human Coronary Atherosclerotic Plaques

\author{
Implications for the Mode of Progression of Advanced Coronary Atherosclerosis
}

\author{
Kevin D. O'Brien, " Margaret D. Allen, ${ }^{\star}$ Thomas O. McDonald, Alan Chait, " John M. Harlan, * Daniel Fishbein, * \\ John McCarty," Marina Ferguson," Kelly Hudkins," Christopher D. Benjamin," Roy Lobb," and Charles E. Alpers" \\ Departments of ${ }^{*}$ Medicine, ${ }^{\ddagger}$ Surgery, and ${ }^{\S}$ Pathology, University of Washington, Seattle, Washington 98195 ; \\ and "Biogen, Inc., Cambridge, Massachusetts 02142
}

\begin{abstract}
Endothelial attachment is the initial step in leukocyte recruitment into developing atherosclerotic lesions. To determine whether vascular cell adhesion molecule-1 (VCAM-1) expression may play a role in inflammatory cell recruitment into human atherosclerotic lesions, immunohistochemistry was performed with a polyclonal rabbit antisera, raised against recombinant human VCAM-1, on 24 atherosclerotic coronary plaques and 11 control coronary segments with nonatherosclerotic diffuse intimal thickening from 10 patients. Immunophenotyping was performed on adjacent sections to identify smooth muscle cells, macrophages, and endothelial cells. To confirm VCAM-1-expressing cell types, double immunostaining with VCAM-1 antisera and each of the cell-specific markers and in situ hybridization were performed.

All atherosclerotic plaques contained some VCAM-1, compared to $45 \%$ of control segments. VCAM-1 was found infrequently on endothelial cells at the arterial lumen in both plaques $(21 \%)$ and in control segments $(27 \%)$, but was prevalent in areas of neovascularization and inflammatory infiltrate in the base of plaques. Double immunostaining and in situ hybridization confirmed that most VCAM-1 was expressed by subsets of plaque smooth muscle cells and macrophages. The results document the presence of VCAM-1 in human atherosclerosis, demonstrate VCAM-1 expression by human smooth muscle cells in vivo, and suggest that intimal neovasculature may be an important site of inflammatory cell recruitment into advanced coronary lesions. (J. Clin. Invest. 1993.92:945-951.) Key words: immunohistochemistry $\bullet$ in situ hybridization • smooth muscle cell $\bullet$ macrophage $\bullet$ endothelial cell
\end{abstract}

\section{Introduction}

Gerrity has demonstrated (1) and Fagiotto and Ross (2, 3) have confirmed that attachment of mononuclear cells to vascular endothelium is among the first steps in the initiation of atherosclerosis. Vascular cell adhesion molecule-1 (VCAM-

This work was presented in part at the 65th Scientific Session of the American Heart Association, 17 November 1992, New Orleans, LA.

Address correspondence to Kevin D. O'Brien, M.D., Division of Cardiology, RG-22, Department of Medicine, University of Washington, Seattle, WA 98195.

Received for publication 14 December 1992 and in revised form 12 March 1993.

J. Clin. Invest.

(C) The American Society for Clinical Investigation, Inc. 0021-9738/93/08/0955/07 \$2.00

Volume 92, August 1993, 945-951
1) ${ }^{1}$ is an inducible molecule that has been shown to mediate endothelial adhesion of monocytes and T lymphocytes (4), the principal leukocyte subsets populating atherosclerotic lesions (3), raising the possibility that VCAM-1 may play a role in the recruitment of inflammatory cells into developing atherosclerotic plaques. Evidence supporting this possibility comes from the demonstration of VCAM immunoreactivity on arterial endothelial cells overlying fatty streaks in Watanabe heritable hyperlipidemic rabbits (WHHL) (5). Although two recent reports have described increased immunoreactivity for intercellular adhesion molecule-1 (ICAM-1) at the arterial luminal surface of atherosclerotic lesions $(6,7)$, the presence or location of VCAM-1 has not been demonstrated in human atherosclerotic lesions to date.

The present study was undertaken to determine whether VCAM- 1 is expressed in human coronary atherosclerosis by performing immunocytochemical studies on human coronary arteries using a rabbit polyclonal antisera raised against recombinant human VCAM-1. To determine which cell types express VCAM-1 in human atherosclerotic plaques, immunohistochemical studies were performed with monoclonal antibodies to identify smooth muscle cells (SMC) and macrophages, and with the lectin, Ulex europaeus I, to distinguish endothelial cells. In situ hybridization using an antisense riboprobe for VCAM-1 also was performed for further confirmation of VCAM-1 expression.

\section{Methods}

\section{Human coronary arterial tissue}

A total of 35 coronary artery segments obtained from 10 hearts explanted at the time of cardiac transplantation were placed in methanolCarnoy's solution (60\% methanol, $30 \%$ chloroform, $10 \%$ acetic acid) within $2 \mathrm{~h}$ of organ excision, fixed for at least $12 \mathrm{~h}$, and then processed and paraffin-embedded according to conventional techniques. 5 of the 10 patients had cardiomyopathy due to atherosclerotic coronary artery disease and the other 5 had idiopathic cardiomyopathy. 6- $\mu \mathrm{m}$ sections were used for immunocytochemical analysis. The 35 coronary artery segments were classified according to conventional histologic criteria into $(a)$ atherosclerotic coronary segments, defined by the presence of typical features of luminal narrowing due to regional accumulation of cholesterol, foam cell, and non-foam cell macrophages, and the presence of fibrous caps, or $(b)$ control coronary segments with diffuse intimal thickening consisting of intimal smooth muscle cells and matrix and representing the characteristic morphology of nonatherosclerotic adult human coronary arteries $(8)$. Additional coronary segments which had been fixed in $10 \%$ neutral buffered formalin (NBF) were

1. Abbreviations used in this paper: $\mathrm{CHO}$, Chinese hamster ovary; ICAM-1, intercellular adhesion molecule-1; NBF, neutral buffered formalin; VCAM-1, vascular cell adhesion molecule-1. 
processed and sectioned in identical fashion and used for in situ hybridization.

\section{Immunohistochemical reagents}

Polyclonal VCAM-1 antisera. Polyclonal antisera directed against VCAM-1 was generated in rabbits as described previously (9). Sensitivity and specificity of the VCAM-1 antisera were determined by the following criteria: (a) positive immunohistochemical staining by the VCAM-1 antisera of VCAM-1-transfected but not of either ICAM-1transfected or untransfected Chinese hamster ovary ( $\mathrm{CHO}$ ) cells; $(b)$ staining of cells in fixed tissue sections known to express VCAM-1, including dendritic cells in lymphoid follicles of human tonsil and parietal epithelial cells of human kidney; and $(c)$ abolition of positive tissue staining on human tonsil and on human coronary atherosclerotic plaques by preincubation of VCAM-1 antisera with VCAM-1-transfected $\mathrm{CHO}$ cells but not by preincubation of the antisera with untransfected or ICAM-1-transfected CHO cells.

Monoclonal antibodies. Immunophenotypic characterization was performed using the following commercially available antibodies: anti-smooth muscle $\alpha$-actin (10) (Dako Corp., Carpinteria, CA), which in this context is specific for SMC and anti-CD68 (Dako Corp.), which recognizes macrophages (9).

Lectins. Ulex europaeus I, a lectin which binds to the fucose moiety and thereby recognizes endothelial cells, was used as described previously $(11,12)$.

\section{Single label immunohistochemistry}

Single label immunohistochemistry was performed as described previously (13), using rabbit VCAM-1 antisera at a titer of 1:2,000, and mouse monoclonal antibodies or lectins at the following titers: antismooth muscle $\alpha$-actin, 1:1,000; anti-CD68, 1:1,000, and Ulex agglutinin, 1:1,000. Briefly, tissue sections were deparaffinized with xylene and then rehydrated with graded alcohols. The slides were blocked with $3 \%$ hydrogen peroxide, washed with PBS, incubated for $60 \mathrm{~min}$ with the primary antibody or lectin, and then washed again with PBS. A biotin-labeled secondary antibody, either anti-rabbit (for VCAM-1 antisera), anti-mouse (for anti-smooth muscle $\alpha$-actin or anti-CD 68), or anti-Ulex, then was applied for $30 \mathrm{~min}$, followed by an avidin-biotin-peroxidase conjugate (ABC Elite; Vector Laboratories, Burlingame, CA) for $30 \mathrm{~min}$. Standard peroxidase enzyme substrate, 3,3'diaminobenzidine with nickel chloride then was added to yield a black reaction product. Cell nuclei were counterstained with methyl green. Negative controls included substitution of primary antisera/antibody with either PBS or irrelevant antibodies to abolish staining.

\section{Double label immunohistochemistry}

Tissue sections were deparaffinized and rehydrated in graded alcohols, then incubated overnight at $4^{\circ} \mathrm{C}$ with VCAM-1 antisera diluted 1:500 in PBS with $1 \%$ BSA. After washing, sections were incubated with goat anti-rabbit IgG with $5 \mathrm{~nm}$ gold (Amersham Corp., Arlington Heights, IL) diluted in PBS $(1 / 40)$ plus $1 \%$ BSA and $0.1 \%$ gelatin for $1 \mathrm{~h}$ at room temperature. Sections were washed and the gold visualized with an IntenSE-M silver enhancement kit (Amersham Corp.). The sections then were incubated sequentially with: $(a)$ anti-smooth muscle $\alpha$-actin, anti-CD68, or Ulex agglutinin, $(b)$ biotinylated horse antimouse IgG or anti-Ulex IgG (Vector Laboratories), and (c) avidin-biotin-alkaline phosphatase complex (Vector Laboratories). The alkaline phosphatase was developed with a red substrate kit (Vector Laboratories) and cell nuclei were counterstained with methyl green. Negative controls included substitution of VCAM-1 antisera which had been preadsorbed against VCAM-1-positive CHO cells for the VCAM-1 antisera, and substitution of isotype-matched normal mouse IgG for the anti-smooth muscle $\alpha$-actin and anti-CD68.

Preparation of Chinese hamster ovary cells for immunohistochemical analysis or in situ hybridization

CHO cells transfected with VCAM-1 were cultured as described previously (9). For use as negative controls, untransfected $\mathrm{CHO}$ cells and
ICAM-1-transfected CHO cells also were cultured. Transfected cells were shown to express either VCAM-1 or ICAM-1 by immunohistochemical analysis and by cell binding assays which were inhibitable by anti-VCAM-1 and anti-ICAM-1 antibodies. Cells were pelleted, fixed in methanol-Carnoy's solution, and paraffin embedded. $6-\mu \mathrm{m}$ sections were cut and prepared for immunohistochemical analysis as described above. Additional cell pellets were fixed in NBF and parrafin embedded, with 6- $\mu \mathrm{m}$ sections used for in situ hybridization.

\section{Adsorption assay}

The VCAM-1 polyclonal antisera was adsorbed against transfected CHO cells as described previously (9). Briefly, cell pellets of $\sim 2.5$ $\times 10^{7}$ cells of VCAM-1-, ICAM-1-, or untransfected cells were collected in PBS and incubated at room temperature for $30 \mathrm{~min}$ with the VCAM- 1 antisera. The suspensions then were centrifuged at $1,200 \mathrm{rpm}$ in a table-top centrifuge and the supernatants collected for incubation on VCAM-1 - and ICAM-1-transfected and untransfected CHO cells, as well as on methanol Carnoy's-fixed human tonsil or coronary arteries. Immunohistochemistry then was performed by standard avidinbiotin immunoperoxidase technique as described above, and cell nuclei counterstained with methyl green.

\section{Riboprobe preparation}

$1 \mu \mathrm{g}$ of a $1.3-\mathrm{kb}$ fragment of the human VCAM-1 gene, including $0.4 \mathrm{~kb}$ of the 3'-untranslated region, in the expression vector pBS (Stratagene Inc., La Jolla, CA) was transcribed into an antisense riboprobe using T3 polymerase, as described previously (13), using $250 \mu \mathrm{Ci}^{35} \mathrm{~S}-\mathrm{UTP}$ (New England Nuclear, Boston, MA) as the radioactive label. After 60 min incubation at $37^{\circ} \mathrm{C}$, the cDNA was digested by adding $1 \mathrm{U}$ RQ 1 DNase (Promega Corp., Madison, WI) and the reaction mixture then was incubated at $37^{\circ} \mathrm{C}$ for an additional $15 \mathrm{~min}$. Free nucleotides were separated using a Sephadex G-50 (Pharmacia LKB, Uppsala, Sweden) column. A sense riboprobe was also transcribed for control hybridizations from a 1.25-kb fragment of the human VCAM-1 gene using T7 polymerase. Probes were stored at $-70^{\circ} \mathrm{C}$ and used within $7 \mathrm{~d}$ of synthesis.

\section{In situ hybridization}

Arterial tissue sections which had been fixed in NBF and embedded in paraffin were deparaffinized according to standard protocol. In situ hybridization was then performed on adjacent sections using the antisense and sense (control) VCAM-1 riboprobes as previously described (13). After the tissue was air dried, it was dipped in NTB2 nuclear emulsion (Kodak) and exposed in the dark at $4^{\circ} \mathrm{C}$ for 2 wk. Control hybridizations also were performed with the antisense VCAM-1 riboprobe on cell pellets of both VCAM-1-transfected and untransfected CHO cells. After developing, sections were counterstained with hematoxylin and eosin.

\section{Results}

\section{Immunohistochemistry}

All coronary arteries with advanced atherosclerotic lesions demonstrated one or more of three identifiable patterns of VCAM-1 expression in coronary artery segments, namely, VCAM-1 expression in association with: $(a)$ arterial luminal endothelial cells, $(b)$ intimal neovasculature, and $(c)$ nonendothelial cells. In most cases this expression was widespread. In contrast, only $45 \%$ of control segments contained any VCAM1 expression, which was sparse, and which was localized to intimal endothelial and nonendothelial cells. Table I details the percentage of segments with any VCAM-1 expression and with each of the three patterns of staining: localization to endothelial cells at the arterial lumen, expression on intimal neovasculature, and expression by nonendothelial cells in the intima.

VCAM-1 expression on arterial endothelium. Similar proportions of plaques and control segments ( 21 and $27 \%$, respec- 
Table I. Distribution of VCAM-1 Expression in Intima

\begin{tabular}{lcccc}
\hline & \multicolumn{3}{c}{ No. positive segments (\%) } & \\
\cline { 2 - 4 } & $\begin{array}{c}\text { Any } \\
\text { VCAM-1 }\end{array}$ & $\begin{array}{c}\text { Arterial luminal } \\
\text { endothelial cell }\end{array}$ & $\begin{array}{c}\text { Neovasculature } \\
\text { cell }\end{array}$ \\
\hline Plaques $(n=24)$ & $24(100)$ & $5(21)$ & $17(71)$ & $22(92)$ \\
Control $(n=11)$ & $5(45)$ & $3(27)$ & $0(0)$ & $2(18)$ \\
\hline
\end{tabular}

The prevalence of any VCAM- 1 expression and of each of three different staining patterns for VCAM- 1 are compared in 24 plaques and 11 control coronary segments. Numbers of positive segments in the group of plaques or controls is given, with percentages in parentheses. Some VCAM-1 staining was found in all plaques examined as compared to $45 \%$ of controls (first column). Of the three different staining patterns, staining at the arterial luminal surface (second column) was the least characteristic of plaques, being found in equal proportions of plaques and controls. Demonstration of VCAM-1 in association with intimal neovasculature was much more characteristic of plaques (third column) and expression by nonendothelial cells was the most common pattern of VCAM-1 expression found in plaques (fourth column).

tively) contained at least some endothelial cells with VCAM-1 expression at the arterial lumen. However, in both groups, the number of arterial endothelial cells with positive VCAM-1 staining was very low. In a single case from the atherosclerosis group, VCAM-1 expression at the arterial lumen was found to occur in an area with foam cell infiltration immediately below the arterial endothelial cell layer (Fig. $1 a$ ). Staining of an adjacent section with Ulex agglutinin (Fig. $1 b$ ) identified these VCAM-1 expressing cells as endothelial cells.

Detection of VCAM-1 in association with neovasculature and nonendothelial cells. While VCAM-1 expression on arterial luminal endothelial cells was equally prevalent in plaques and control segments, the other two staining patterns, that is, VCAM- 1 expression in association with intimal neovasculature and in association with nonendothelial cells in the intima, were much more prevalent in plaques than in control segments (Table I). VCAM-1 expression was present in at least some intimal neovessels in the majority $(71 \%)$ of plaques. Only one control segment contained any intimal neovasculature and that coronary segment did not have VCAM-1 expression. Thus, neovascular VCAM-1 expression was a much more prevalent finding in plaques than was arterial luminal endothelial cell VCAM-1 expression.

The most striking finding of this study was the demonstration of VCAM-1 expression by nonendothelial cells, which was the most prevalent pattern of VCAM-1 expression in both plaques and control segments. Nonendothelial cell VCAM-1 expression was identified in $92 \%$ of plaques but only $18 \%$ of control segments ( Table I). Fig. 2 demonstrates these two additional patterns of VCAM-1 expression. Fig. $2 a$ shows a low power view of the lower intima (top) and upper media (bottom) of a hematoxylin and eosin-stained plaque, with a black arrow identifying the internal elastic lamina. Fig. $2, b-d$, shows higher power views of the central portion of Fig. $2 a$, which contains neovascular ingrowth and increased cellularity, with a black arrow identifying the internal elastic lamina. The VCAM- 1 antisera (Fig. $2 b$ ) demonstrates that VCAM- 1 is present in association with the neovasculature. An adjacent section stained with Ulex (Fig. $2 c$ ) confirms the presence of endothe- lial cells in VCAM-1-positive neovasculature. However, VCAM-1 staining (Fig. $2 b$ ) also can be seen in association with nonendothelial cells in the base of the plaque and in the upper portion of the media. Areas which contained nonendothelial cell VCAM-1 expression tended to have associated inflammatory cell infiltrate, as demonstrated with the macrophage-specific antibody, anti-CD68 (Fig. $2 d$ ). Colocalization studies demonstrated that the majority of VCAM-1 expression in the arterial intima is found in a subset of intimal SMC, but that occasional VCAM-1-positive staining could also be detected on SMC in the upper media (Fig. $2 b$ ) and on subsets of plaque macrophages, including both non-foam cell and foam cell macrophage phenotypes.

Adventitial VCAM-1 expression and comparison with neovascular VCAM-1. Because VCAM-1 expression has been documented in association with adventitial endothelial cells (14), its presence or absence in adventitial vessels was determined by immunocytochemistry with the VCAM-1 antisera in all 35 segments in this study. As shown in Table II, VCAM-1 expression was present in adventitial vessels in the majority of arterial segments with plaques $(87.5 \%)$, but only in a minority of control coronary segments $(27 \%)$. The relationship between plaque adventitial and neovascular VCAM-1 expression also was evaluated, as shown in Table III. The majority of plaques had both adventitial and neovascular VCAM-1 expression $(62.5 \%)$; in only one case was neovascular VCAM-1 expression detected in a plaque without adventitial VCAM-1. However, though VCAM-1 expression was seen in both adventitial vessels and neovasculature in many plaques, adventitial vessel VCAM-1 expression in one region of the plaque was not necessarily associated with VCAM-1 expression in contiguous neovasculature. Likewise, VCAM-1 expression by neovasculature was not necessarily associated with VCAM-1 expression on underlying adventitial vessels.

\section{Double label immunohistochemistry}

To definitively determine which nonendothelial cell types expressed VCAM-1, double label immunohistochemistry was performed with the VCAM-1 antisera and with either antismooth muscle $\alpha$-actin or anti-CD68 antibodies (Fig. 3). Cellspecific antibodies were identified with an alkaline phosphatase method and appear red on slides, while the VCAM-1 antisera was marked with an immunogold technique and appears black. Double label immunohistochemistry with anti-smooth muscle $\alpha$-actin and VCAM-1 antisera confirmed that VCAM-1 expression by nonendothelial cells in the intima was primarily

Table II. Adventitial VCAM-1 Expression in Plaques and Control Coronary Segments

\begin{tabular}{llcc}
\hline & & \multicolumn{2}{c}{ No. of segments (\%) } \\
\cline { 3 - 4 } & & Plaque & Controls \\
\hline Adventitial & Present & $21(87.5)$ & $3(27)$ \\
VCAM-1 & Absent & $3(12.5)$ & $8(73)$ \\
& Total & $24(100)$ & $11(100)$
\end{tabular}

Adventitial VCAM-1 expression as detected by immunocytochemistry was much more prevalent in plaques than in control coronary segments, being detected in $21 / 24(87.5 \%)$ of plaques as compared to only $3 / 11(27 \%)$ of control coronary segments. 

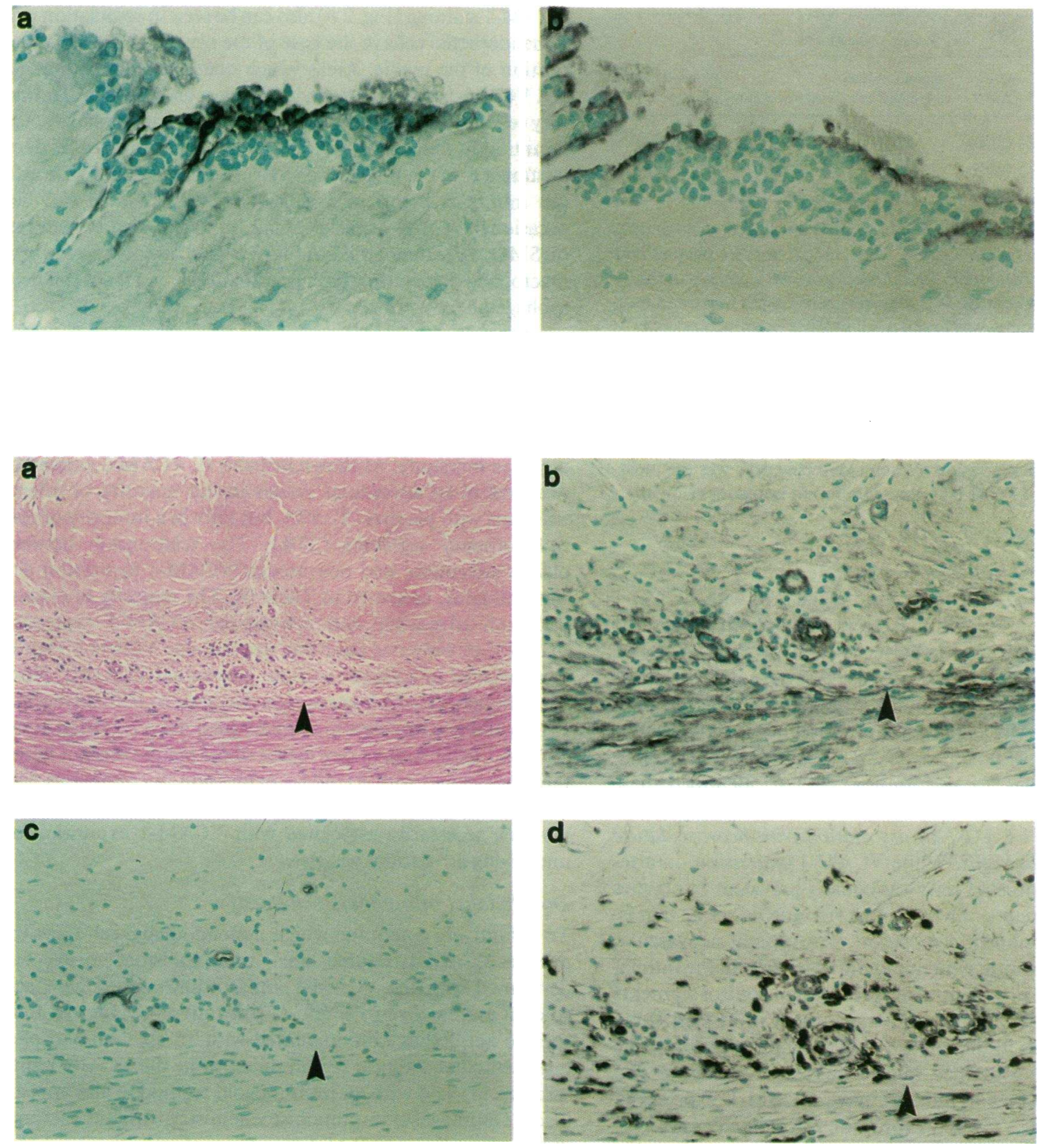

Figure 1. VCAM-1 Expression by arterial luminal endothelial cells. Staining with the VCAM-1 antisera (black reaction product) is present along the luminal surface of an artery overlying an inflammatory cell infiltrate $(a)$. Staining of an adjacent section with the Ulex lectin $(b)$ identifies the VCAM-1-positive cells in $a$ as endothelial cells (black reaction product). (Methyl green counterstain, final magnification, 872).

Figure 2. VCAM-1 Expression on intimal neovasculature and nonendothelial cells. A low power view of another plaque demonstrates the presence of neovascular ingrowth and increased cellularity in the base of the plaque $(a)$. Higher power views of adjacent sections stained with VCAM-1 antisera $(b)$, Ulex lectin $(c)$, and anti-CD68 $(d)$ are developed so that each marker is identified by a black reaction product. A black arrow identifies the internal elastic lamina in each photomicrograph. VCAM-1 is expressed on neovasculature $(b)$ with intact endothelial cells $(c)$. VCAM-1 is also expressed by nonendothelial cells in the intima and upper media $(b)$. Neovascular and nonendothelial cell VCAM-1 expression generally occurred in areas of inflammatory cell infiltrate, as demonstrated by the presence of macrophages in this region $(d)$. $(a)$ hematoxylin and eosin counterstain, final magnification, 436; $b-d$; methyl green counterstain, final magnification, 872).

associated with a subset of intimal SMC (Fig. $3 a$ ). Positive staining with the VCAM-1 antisera could be abolished by preincubation with VCAM-1-expressing CHO cells (Fig. $3 b$ ). Occasional intimal macrophages also could be shown to express VCAM-1 by double label immunohistochemistry with anti-CD68 and VCAM-1 antisera (Fig. $3 c$ ).
VCAM-1 expression by endothelial cells was often quite discrete. Fig. $3 d$ demonstrates double label immunocytochemistry in an adventitial vessel with both the VCAM-1 antisera and anti-smooth muscle $\alpha$-actin. In this example, some endothelial cells were positive for VCAM-1 while others were negative. Preservation of an endothelial cell lining covering the 
Table III. Comparison of Plaque Neovascular and Adventitial VCAM-1 Expression

\begin{tabular}{llclr}
\hline & \multicolumn{3}{c}{ No. of segments (\%) } \\
\cline { 3 - 4 } & & \multicolumn{2}{c}{ Neovascular VCAM-1 } \\
\cline { 3 - 5 } & & Present & Absent \\
\hline Adventitial & Present & $15(62.5)$ & $6(25)$ & $21(87.5)$ \\
VCAM-1 & Absent & $1(4.2)$ & $2(8.3)$ & $3(12.5)$ \\
& & $16(66.7)$ & $8(33.3)$ & $24(100)$
\end{tabular}

Some expression of VCAM-1 by both adventitial vessels and by neovasculature was detected in the majority of plaques $(15 / 24$ or $62.5 \%)$, and the majority of plaques with VCAM-1-positive neovasculature had adventitial VCAM-1 expression ( $15 / 16$ or $93.8 \%)$. However, evaluation of individual plaques indicated that neovascular VCAM-1 could be seen in the absence of VCAM-1 expression by underlying adventitia.

whole circumference of the lumen of this adventitial vessel was confirmed by positive Ulex staining of an adjacent tissue section. This discrete expression of VCAM-1 by adventitial as well as neovascular and arterial luminal endothelial cells is consistent with local, rather than systemic, control of VCAM-1 expression.

\section{In situ hybridization}

To further confirm that nonendothelial cells in human coronary arteries express VCAM-1, in situ hybridization for VCAM-1 was performed on additional coronary artery segments using a ${ }^{35} \mathrm{~S}$-labeled antisense riboprobe transcribed from a 1.3-kb fragment of the human VCAM-1 gene. Segments used for in situ hybridization were fixed in NBF rather than in methanol Carnoy's solution, as was used for immunohistochemistry. These experiments confirmed that endothelial cells, macrophages, and SMC in human coronary artery segments express VCAM-1 mRNA. VCAM-1 mRNA expression by intimal smooth muscle cells is demonstrated in Fig. 4. Control hybridizations performed on adjacent arterial sections using a sense riboprobe demonstrated the absence of specific hybridization (data not shown). Additional control experiments performed using the antisense VCAM-1 riboprobe showed positive in situ hybridization only with NBF-fixed cell pellets of VCAM-1-transfected CHO cells, and not with similarly fixed untransfected $\mathrm{CHO}$ cells.

\section{Discussion}

VCAM-1 expression by endothelial cells is believed to mediate attachment of mononuclear cells that constitutively express the very late activation antigen-4 (VLA-4) receptor, i.e., monocytes and lymphocytes (4). Because these are the inflammatory cell subsets typically identified in human coronary plaques, it has been proposed that VCAM-1 may play an important role in their recruitment into developing plaques $(5$, 15 ). The present study establishes that cells expressing VCAM1 are present in advanced human coronary atherosclerosis. The majority of these cells were found in the deeper portions of plaque, in areas of inflammation and neovascularization, as compared to endothelial cells at the arterial lumen. Perhaps the most surprising finding was that although VCAM-1 sometimes was found in association with arterial and neovascular endothelial cells, the majority of VCAM-1 expression in plaques occurred in subsets of intimal SMC and macrophages.

Arterial endothelial cell reactivity with the VCAM-1 antisera in human plaques corresponded in appearance to the pattern of VCAM immunoreactivity which has been reported by others in fatty streaks of WHHL rabbits (5). However, this was an infrequent finding in the present study, possibly because the atherosclerotic segments examined generally contained advanced lesions rather than fatty streaks. Instead, endothelial cell expression of VCAM-1 was most prevalent in areas of neovascularization, usually in association with an adjacent inflammatory cell infiltrate. It has been thought that the majority of inflammatory cells entering the plaque are recruited from the arterial lumen and that plaques go through an orderly development from fatty streak, consisting primarily of macrophages which have accumulated lipid $(1,2)$, to a fibrofatty lesion, in which smooth muscle cells are recruited from the media to form a "cap" over a core composed of the inflammatory infiltrate and lipid, and finally to a fully developed fibrous lesion, in which the inflammatory core of the lesion has largely resolved (3). According to that hypothesis, further plaque growth may occur in part by infiltration of more macrophages from the arterial lumen and formation of another fibrous cap (3).

This study demonstrates that there is significant expression in the base of plaques of a molecule that may recruit inflammatory cells. This finding suggests that inflammatory cells may enter the plaque from the vasa vasorum of the arterial media and adventitia. Intimal neovascularization, which occurs by ingrowth of the vasa vasorum into the base of the plaque, thus may provide a further route for inflammatory cell entry into the base of the intima. The stimuli that mediate the ingrowth of the neovasculature present in the base of plaques are not known. In this study, intimal neovascularization was a frequent occurrence, having been identified by histology and confirmed by Ulex staining of endothelial cells in $22 / 24$ of the advanced coronary plaques examined. The expression of VCAM- 1 is indicative that the neovascularization process is associated with assumption of an "activated" state by the endothelial cells (16). This state is likely to be biologically signifcant, as indicated by the frequent presence of inflammatory cell infiltrate around these neovessels.

VCAM-1 expression by adventitial endothelium also was highly specific for arterial segments involved by plaque, being present in $87.5 \%$ of these specimens as compared to $27 \%$ of control segments. Also, 15 of $16(93.8 \%)$ plaques with neovascular VCAM-1 expression had adventitial VCAM-1 expression. Thus, expression of VCAM-1 in adventitial vessels and in neovasculature are much more characteristic of plaques than control segments, raising the possibility that factors associated with atherosclerosis may upregulate endothelial cell VCAM-1 expression in both the adventitia and the intima. Several findings suggest that VCAM-1 expression in both adventitia and neovasculature is under local control. First, VCAM-1 was not expressed by every adventitial vessel nor by every neovessel in any specimen examined, so that its expression in plaques did not appear to be constitutive. Second, VCAM-1 expression by adventitial vessels in a particular region of a plaque was not necessarily associated with VCAM-1 expression on adjacent neovasculature, consistent with the hypothesis that expression by contiguous adventitial vessels is not a necessary precursor for neovascular VCAM-1 expression. Finally, as demonstrated 

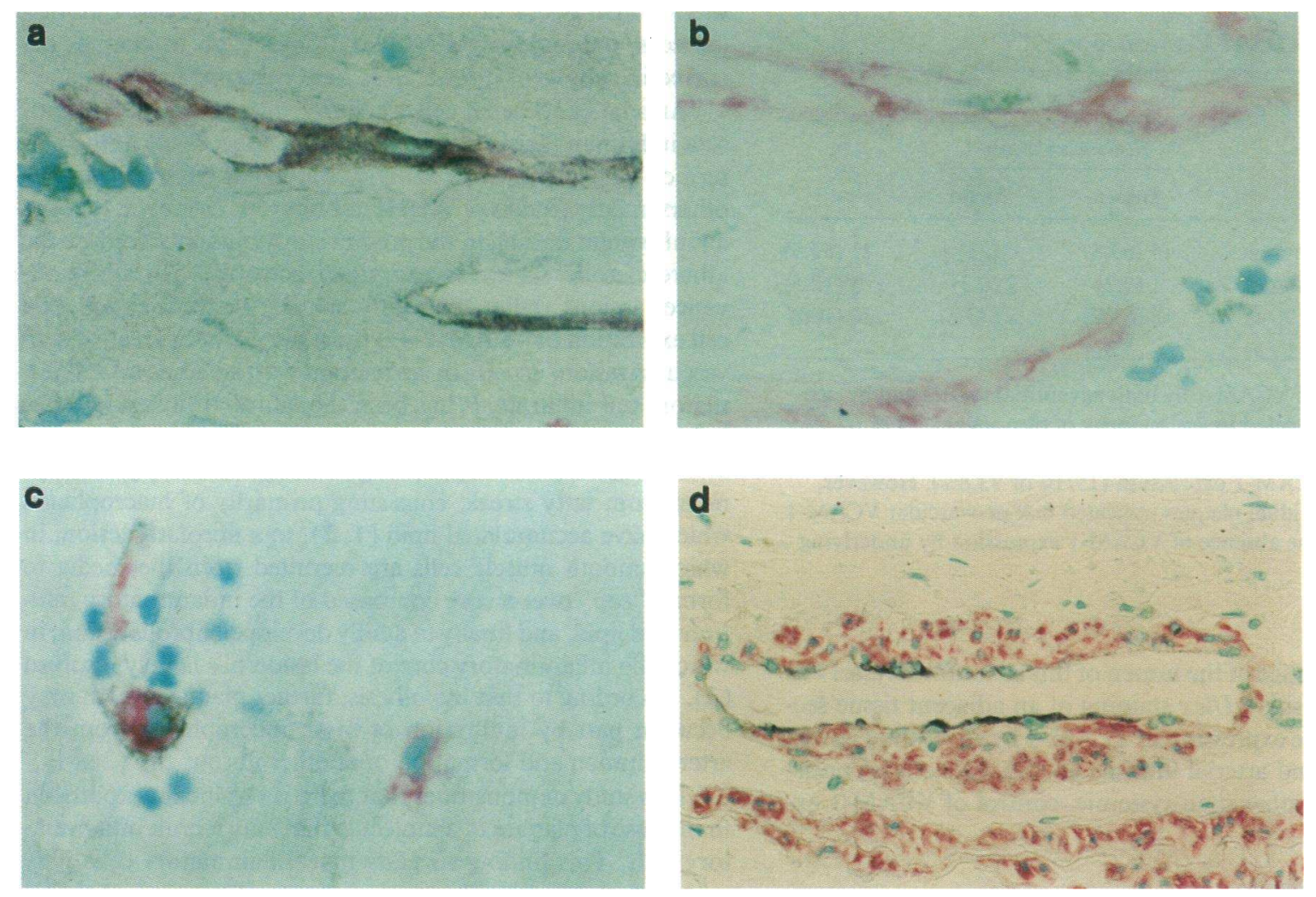

Figure 3. Double immunostaining with VCAM-1 antisera and nonendothelial cell markers and abolition of VCAM-1 staining by preincubation with VCAM-1-expressing CHO cells. VCAM-1 antisera ( $a$, $c$, and $d$ ) or VCAM-1 antisera preadsorbed against VCAM-1-expressing $\mathrm{CHO}$ cells $(b)$ was developed with an immunogold technique to yield a black reaction product, while the cell specific markers, anti-smooth muscle $\alpha$-actin $(a, b$, and $d)$ and anti-CD68 $(c)$, were developed with an alkaline phosphatase technique to yield a red reaction product. The VCAM-1 antisera detects VCAM-1 on cells identified as smooth muscle cells $(a)$. Preadsorption of the VCAM-1 antisera with VCAM-1-expressing CHO cells abolishes staining $(b)$, confirming the specificity of the antisera for VCAM-1. VCAM-1 expression also is identified on cells which are positive for the macrophage marker anti-CD68 $(c)$. While VCAM-1 expression was detectable on some endothelial cells, adjacent endothelial cells could be negative, as shown in an adventitial vessel $(d)$ (methyl green counterstain, final magnification, 2,178).

Figure 4. In situ hybridization for VCAM-1. Black autoradiography grains identifying the presence of VCAM-1 mRNA are detectable in spindled mesenchymal cells. (Hematoxylin and eosin counterstain, final magnification, 1,452).

in Fig. $3 d$, single endothelial cells could be found which expressed VCAM-1, while adjacent endothelial cells did not, again consistent with the hypothesis that most VCAM-1 expression in adventitia and neovasculature is controlled by local factors.

The observation that most VCAM-1 in atherosclerotic plaques is found on SMC and, less commonly, on macrophages rather than on endothelial cells is somewhat surprising but not unprecedented. Detection of VCAM-1 expression by nonendothelial cells has been described in a variety of other settings, including dendritic cells in human lymphoid tissue (14) and in cutaneous graft-versus-host disease (17). Renal glomerular epi- thelial and tubular cells, some of mesenchymal origin, also have been shown to express VCAM-1 under certain circumstances (14). Cultured bone marrow-derived fibroblasts recently have been shown by flow cytometry and immunocytochemistry to express VCAM-1 (18), and TNF- $\alpha$-stimulated neural cells also express this molecule (19). Recently, expression of VCAM-1 on secondary myoblasts during myogenesis has been described (20), indicating that, under certain conditions, muscle cells may express VCAM-1. In this study we demonstrate, by immunohistochemistry and by in situ hybridization, VCAM-1 expression on neointimal SMC, but not generally on the population of SMC in the media of the vessel wall. 
Because association of VCAM-1 with intimal SMC usually was found in areas of inflammatory cell infiltrate, this suggests that some factors related to inflammation (e.g., release of various cytokines) may control expression of VCAM-1 by intimal SMC. How VCAM-1 expression by SMC in atherosclerotic plaques may promote the process of atherogenesis is unknown.

In the present study, it also was determined that a subset of intimal macrophages expressed VCAM-1 as assessed by double immunostaining with VCAM- 1 antisera and the anti-macrophage monoclonal antibody, anti-CD68, and as confirmed by in situ hybridization. It is noteworthy that VCAM-1 expression recently has been demonstrated in association with macrophages in rheumatoid synovium, but not in synovium from patients with osteoarthritis (21). Thus, specific cytokines that are expressed in the inflammatory state of rheumatoid synovium and induce VCAM- 1 expression in macrophages also may regulate its expression by subsets of human coronary intimal macrophages.

Recruitment by VCAM-1 of inflammatory cells into the base of plaques could have important adverse effects on plaque stability. Macrophages may secrete many proteolytic enzymes, including collagenase and stromelysin, which may weaken the plaque and increase its propensity to rupture (22). Elegant computer modeling also has been used to demonstrate that the presence of a soft "load-bearing surface" in the base of plaques, as could be created by the infiltration of proteolytic enzyme-releasing inflammatory cells, could increase the mechanical stress on fibrous caps, thereby increasing their propensity to rupture (23). However, there is not yet sufficient information to conclude that areas of leukocytic aggregation in atherosclerosis have a greater predilection for rupture than other regions within the plaque.

In summary, the present study demonstrates the presence of VCAM-1 in advanced human coronary atherosclerotic plaques, suggesting a role for VCAM-1 expression in the recruitment of inflammatory cells into plaques. VCAM-1 in association with arterial luminal endothelial cells was infrequent and VCAM-1 in association with neovasculature in the base of plaques more prevalent, suggesting that the intimal neovasculature may be an important site of continued inflammatory cell recruitment into plaques after initiation. VCAM-1 expression was demonstrated on SMC in the neointima. Although the functional significance of this last finding cannot be assessed as yet, it does suggest that, in addition to mediating inflammatory cell attachment to endothelium, VCAM-1 expression reflects an "activated" state of specific cell types important in atherogenesis. This can be considered analogous to the upregulated expression of Class II MHC molecules by these SMC in atherosclerosis that has been reported by others $(16,24)$, and further supports the concept that, at least in part, atherosclerosis is a form of immune injury to the arterial wall.

\section{Acknowledgments}

The authors thank Elaine Yamanaka for expert technical assistance, Lisa Anne Billings for help in preparing the manuscript, and Dr. B. Greg Brown for constructive comments. The authors also gratefully acknowledge the contributions of Dr. Salim Aziz of the University of Washington Cardiac Transplantation Program.

This work was supported in part by National Institutes of Health Grants HL-42270, HL-47151, HL-18645, and HL-30086, and in part by a grant from the Pacific Foundation for Cardiovascular Research.
Dr. O'Brien was a Research Fellow of the American Heart Association, Washington Affiliate.

\section{References}

1. Gerrity, R. G. 1981. The role of the monocyte in atherogenesis. I. Transition of blood-borne monocytes into foam cells in fatty lesions. Am. J. Pathol 103:181-190.

2. Faggiotto, A., R. Ross, and L. Harker. 1990. Studies of hypercholesterolemia in the nonhuman primate. I. Changes that lead to fatty streak formation. Arteriosclerosis. 4:323-340.

3. Ross, R. 1986. The pathogenesis of atherosclerosis: an update. N. Engl. J. Med. 314:488-500.

4. Springer, T. A. 1990. Adhesion receptors of the immune system. Nature (Lond.). 346:425-434.

5. Cybulsky, M. I., and M. A. Gimbrone, Jr. 1991. Endothelial expression of a mononuclear leukocyte adhesion molecule during atherogenesis. Science (Wash. DC). 251:788-791.

6. Poston, R. N., D. O. Haskard, J. R. Coucher, N. P. Gall, and R. R. JohnsonTidey. 1992. Expression of intercellular adhesion molecule-1 in atherosclerotic plaques. Am. J. Pathol. 140:665-673.

7. Printeseva, O. Y., M. M. Peclo, and A. M. Gown. 1992. Various cell types in human atherosclerotic lesions express ICAM-1. Further immunocytochemical and immunochemical studies employing monoclonal antibody 10F3. Am. J. Pathol. 140:889-896.

8. Gordon, D., M. A. Reidy, E. P. Benditt, and S. M. Schwartz. 1990. Cell proliferation in human coronary arteries. Proc. Natl. Acad. Sci. USA. 87:46004604.

9. Bacchi, C. E., C. L. Marsh, C. D. Perkins, R. L. Carithers, J. P. McVicar, K. L. Hudkins, C. D. Benjamin, J. M. Harlan, R. Lobb, and C. E. Alpers. 1993. Expression of vascular cell adhesion molecule (VCAM-1) in liver and pancreas allograft rejection. Am. J. Pathol. 142:579-591.

10. Skalli, O., P. Ropraz, A. Trzeciak, G. Benzonan, D. Gillessen, and G. Gabbiani. 1986. A monoclonal antibody against $\alpha$-smooth muscle actin: a new probe for smooth muscle differentiation. J. Cell Biol. 103:2787-2796.

11. Holthöfer, H., I. Virtanen, A.-L. Kariniemi, M. Hormia, R. Linder, and A. Miettinen. 1982. Ulex europaeus I lectin as a marker for vascular endothelium in human tissues. Lab. Invest. 47:60-66.

12. Alpers, C. E., and J. H. Beckstead. 1985. Monocyte/macrophage-derived cells in normal and transplanted human kidneys. Clin. Immunol. Immunopathol. 36:129-140.

13. O'Brien, K. D., D. Gordon, S. Deeb, M. Ferguson, and A. Chait. 1992. Lipoprotein lipase is synthesized by macrophage-derived foam cells in human coronary atherosclerotic plaques. J. Clin. Invest. 89:1544-1550.

14. Rice, G. E., J. M. Munro, C. Corless, and M. P. Bevilacqua. 1991. Vascular and nonvascular expression of INCAM-110: a target for mononuclear leukocyte adhesion in normal and inflamed human tissues. Am. J. Pathol. 138:385393.

15. Libby, P., and G. K. Hansson. 1991. Involvement of the immune system in human atherogenesis: current knowledge and unanswered questions. $L a b$. Invest. 64:5-15.

16. Hansson, G. K., L. Jonasson, J. Holm, and L. Claeson-Welch. 1986. Class II MHC antigen expression in the atherosclerotic plaque: smooth muscle cells express HLA-DR, HLA-DQ, and the invariant gamma chain. Clin. Exp. Immunol. 64:261-268.

17. Norton, J., J. P. Sloan, N. Al-Saffar, and D. O. Haskard. 1991. Vessel associated adhesion molecules in normal skin and acute graft-versus-host disease. J. Clin. Pathol. (Lond.). 44:586-591.

18. Ryan, D. H., B. L. Nuccie, C. N. Abboud, and J. M. Winslow. 1991. Vascular cell adhesion molecule-1 and the integrin VLA-4 medicate adhesion of human B cell precursors to cultured bone marrow adherent cells. J. Clin. Invest. 88:995-1004.

19. Birdsall, H. H., C. Lane, M. N. Ramser, and D. C. Anderson. 1992. Induction of VCAM-1 and ICAM-1 on human neural cells and mechanisms of mononuclear leukocyte adherence. J. Immunol. 148:2717-2723.

20. Rosen, G. D., J. R. Sanes, R. LaChance, J. M. Cunningham, J. Roman, and D. C. Dean. 1992. Roles for the integrin VLA-4 and its counter receptor VCAM-1 in myogenesis. Cell. 69:1107-1119.

21. Koch, A. E., J. C. Burrows, G. K. Haines, T. M. Carlos, and J. M. Harlan 1991. Immunolocalization of endothelial and leukocyte adhesion molecules in human rheumatoid and osteoarthritic synovial tissues. Lab. Invest. 64:313-320.

22. Lendon, C. L., M. J. Davies, G. V. R. Born, and P. D. Richardson. 1991. Atherosclerotic plaque caps are locally weakened when macrophages density increases. Atherosclerosis. 87:87-90.

23. Richardson, P. D., M. J. Davies, and G. V. R. Born. 1989. Influence of plaque configuration and stress distribution on fissuring on coronary atherosclerotic plaques. Lancet. 2:941-944.

24. Hansson, G. K., J. Holm, and L. Jonasson. 1989. Detection of activated T lymphocytes in the human atherosclerotic plaque. Am. J. Pathol. 135:169-175. 